\section{Cahiers de Narratologie}

Analyse et théorie narratives

$33 \mid 2018$

L'Art du roman chez Umberto Eco

\title{
La Mystérieuse flamme de la reine Loana et l'espace vide de l'individuel
}

\section{Patrizia Violi}

Translator. Traduit de l'italien par Mohamed Bernoussi

\section{OpenEdition}

\section{Journals}

\section{Electronic version}

URL: http://journals.openedition.org/narratologie/8110

DOI: 10.4000/narratologie.8110

ISSN: $1765-307 X$

Publisher

LIRCES

\section{Electronic reference}

Patrizia Violi, «La Mystérieuse flamme de la reine Loana et l'espace vide de l'individuel », Cahiers de Narratologie [Online], 33 | 2018, Online since 23 July 2018, connection on 15 November 2019. URL : http://journals.openedition.org/narratologie/8110 ; DOI : 10.4000/narratologie.8110

This text was automatically generated on 15 November 2019.

Article L.111-1 du Code de la propriété intellectuelle. 


\title{
La Mystérieuse flamme de la reine Loana et l'espace vide de l'individuel
}

\author{
Patrizia Violi
}

Translation : Traduit de l'italien par Mohamed Bernoussi

\begin{abstract}
«Et de fait ma tête n'était pas vide, y tourbillonnaient des mémoires qui n'étaient pas à moi, la marquise sortit à cinq heures au milieu du chemin de notre vie, Ernesto Sábato je suppose, Abraham engendra Issac, Issac engendra Jacob, Jacob engendra Judas et Rocco ses frères, pour qui le docteur de Chantemerle sonne la minuit sainte et ce fut alors que je vis le pendule, sur ce bras du lac de Côme dorment les oiseaux qui vont mourir au Pérou, messieurs les Anglais je me suis couché de bonne heure, ici on fait l'Italie ou on tue un homme mort, tu quoque alea, et d'un seul coup d'un seul, il lui fend le cœur, frères d'Italie encore un effort, une souris blanche qui siffle sur nos têtes, la valeur n'attend pas, l'Italie est faite mais ne se rend pas, un quarteron de généraux, qu'allait-il faire dans ce Boeing, pas de printemps pour la conscience, le train sifflera avez-vous vu Mirza la cantatrice sur les ailes dorées, mais où sont les neiges d'antan, ô temps suspends ton vol mignonne allons voir si la rose, c'est nous les canuts

(....)

L'encyclopédie me tombait dessus en feuilles éparpillées, et je me mettais à frapper des mains comme au milieu d'un essaim d'abeilles. Pendant ce temps, les enfants disaient grand-père, je savais que j'aurais dû les aimer plus que moi-même et je ne savais pas qui appeler Giorgio, qui Alessandro et qui Luca. Je savais tout sur Alexandre le Grand, et rien d'Alessandro mon tout petit. »

La Mystérieuse flamme de la reine Loana, 2005, p. 24-25)
\end{abstract}

Ce passage est extrait de La Mystérieuse flamme de la reine Loana, roman d'Umberto Eco publié en Italie en 2004. Il me servira à introduire quelques réflexions, non seulement sur le Roman en question, mais aussi sur le rapport entre l'écriture narrative d'Umberto Eco et sa réflexion théorique. Loin d'être deux territoires radicalement séparés, fiction et théorie sont plutôt deux champs complémentaires, liés par un rapport qui, bien que non immédiatement visible, se révèle à un regard plus attentif extrêmement significatif pour reconstruire le sens total de la vision d'Eco. 
2 On pourrait dire que la forme narrative illustre avec une force et une puissance métaphorique tout à fait particulière ce, qui dans les textes théoriques, est expliqué à travers un langage non figuré, plus didactique donc, et peut être moins puissant. Mais il existe aussi une autre raison qui explique le rapport étroit entre travail théorique et création romanesque, peut-être même plus importante. Sur le dos de couverture de la première édition italienne du Nom de La rose, Eco écrivait: "ce que l'on ne peut théoriser on doit le narrer ». Je n'ai pas trouvé de références ultérieures à cette affirmation, mais il n'est sans aucun doute pas infondé de lire les romans d'Eco à contrecourant de sa théorie, comme une sorte de contrechamp qui, loin d'appartenir à un domaine séparé et distinct, instaure un lien profond entre œuvre théorique et œuvre romanesque. La Fiction a pour tâche de faire allusion à ces problèmes que la théorie laisse irrésolus, les évoquant non seulement à travers un langage différent, mais sur un autre plan de pertinence. Les romans d'Umberto Eco remplissent, me semble-t-il, une fonction, que nous pourrions rapprocher de celle des mythes, ainsi que la définissait Claude Lévi-Strauss : selon l'anthropologue français, les mythes mettent en tension des valeurs opposées qu'une culture donnée n'a pas encore réussie à reconstituer en récit unitaire ou cohérent. De façon analogue, les romans d'Eco mettent en scène des problématiques qui n'ont pas trouvé de solution sur le plan théorique, et qui ne la trouveraient peut-être jamais. A ce titre, La Reine Loana n'est pas seulement la meilleure représentation narrative de l'Encyclopédie, ce grand répertoire de savoirs qui dit et conditionne notre être, mais va au cœur d'un autre problème fondamental, en partie irrésolu par la sémiotique d'Eco : la subjectivité individuelle et son irréductible singularité. Le roman est en même temps la représentation de la richesse infinie de l'encyclopédie et des apories qu'une telle notion laisse irrésolues.

3 Mais revenons un moment au roman. Yambo, le protagoniste, à la suite d'un accident non précisé, reste affecté par une singulière forme d'amnésie rétrograde qui envahit toute la sphère de son existence personnelle, laissant en même temps intactes ses très vastes connaissances encyclopédiques. Pour le dire selon les termes du docteur Gratarolo qui le soigne, sa mémoire sémantique est parfaitement fonctionnelle, mais la mémoire autobiographique, elle, est perdue, celle constituée de tous les épisodes, grands et petits, qui forment notre histoire individuelle, unique et irréversible, cette mémoire qui nous permet de dire « je », ou mieux, d'être un " je ». Et en fait, le pauvre Yambo ne se souvient même pas de son nom, qu'il a sur le bout de la langue. Il sait tout ce que les autres savent aussi, tout ce qu'on apprend pour l'avoir lu quelque part, ou par ouï-dire, mais pas ce qui est associé à ses expériences directes.

4 En réalité La situation pathologique de Yambo rappelle singulièrement la position du sujet telle qu'elle a été traitée par Eco, depuis Le Trattato di semiotica generale a Semiotica e filosofia del linguaggio. Le sujet dans la théorie d'Eco est un sujet de type assez particulier, ni transcendantal et encore moins psychologique, mais essentiellement culturel. La subjectivité n'est pas associée à une forme définissable de façon spécifique, mais renvoie plutôt à une constellation diffuse de parcours de sens possibles à l'intérieur de cette encyclopédie générale de toutes les connaissances stratifiées et en métamorphose continue que constitue la semiosis, interminable activité de production du sens. Les sujets sont inscrits dans ce réseau, et existent seulement dans la mesure où ils en représentent les parcours possibles ou, au plus, en préfigurent les transformations. Ce sont des sujets culturels, non psychiques et comme tels 
entièrement réalisés dans la culture qui les forme; leur individualité n'est jamais interrogée à partir d'une mémoire personnelle, d'un parcours singulier.

5 L'énonciation individuelle, loin d'être l'expression d'un jeu transcendantal résultat d'une "schizie créatrice» (Greimas et Courtès 1979: 79) qui se projette dans les énoncés en y laissant ses traces, est plutôt une sorte de modulation du déjà dit ${ }^{1}$, prise dans un renvoi continu à d'autres paroles et à d'autres énonciations, une subjectivité faite des "écumes et débris» de l'encyclopédie (Eco 1984: 54). Le sujet individuel disparaît dans ce réseau et ne détient aucune position centrale et fondamentale, diffus dans le réseau des processus de production des signes, il se fait sujet historique et culturel, non mémoire du singulier ou de l'unique.

6 Là, Yambo apparaît comme la représentation fictive d'un équivalent sujet encyclopédique, un homme sans individualité qui circule avec une parfaite maitrise des savoirs partagés de sa culture, mais qui a perdu le réseau de leurs secrètes et intimes assonances. Il se rend compte que, sans la clef du code de leur propre singularité, l'infini réseau encyclopédique se présente comme un éventail insensé et fou de chaînes associatives privées d'un vrai sens. Ce n'est pas tant la distance historique pacifiée et recomposée que Yambo expérimente dans sa perte de la mémoire, mais plutôt la perte angoissante de sa propre authenticité.

7 Émerge dans cette profonde angoisse une des premières apories théoriques à laquelle je faisais allusion au début. L'encyclopédie culturelle de son temps, à l'intérieure de laquelle Yambo semblait circuler encore avec maestria, semble insuffisante pour restituer le sens profond de sa propre existence. Si la théorie d'Eco fait écho à la maxime antique «de individuo nulla scienza », dans l'œuvre romanesque c'est justement la connaissance individuelle qui réclame ses propres droits. Il est connu qu'Eco a toujours nourri indifférence, sinon doute et méfiance, envers les deux grands courants de pensée du siècle dernier qui ont mis au centre de leur réflexion l'individu: le féminisme et la psychanalyse. Eco ne s'est jamais occupé dans sa théorie de ces savoirs, et, dans certains cas, a explicitement pris distance, mais c'est justement le lieu (vide) de ce savoir qui constitue le pivot narratif et pathémique de La Mystérieuse flamme de la reine Loana. L'amnésie dont souffre Yambo, durant la recherche désespérée d'une mémoire autobiographique et singulière non réductible à la mémoire culturelle et collective, semble représenter, sous une forme spéculaire et inversée, cette absence qui est en revanche fondatrice sur le plan de la théorie sémiotique. Absence revendiquée dans les écrits théoriques comme nécessité interne, mais mise en scène dans les écrits romanesques comme limite douloureuse.

8 Pour retrouver le fil de sa mémoire, et avec lui le sens de sa propre existence, Yambo se retire en effet seul dans la maison de Solara qui a été la maison de son enfance, encore pleine de souvenirs, de livres, de revues, d'objets appartenant à cette époque et qui devraient l'aider à remonter de mot en mot la chaîne associative jusqu'au « mot final ", peut-être cet impossible « je » qui semble se soustraire à toute recherche. Commence ainsi le voyage de Yambo dans la mémoire de son enfance et dans l'histoire de l'Italie fasciste (Yambo comme Eco est né en 1932), et ensuite de la guerre, de la résistance qui arrive jusqu' à Solara, passant par la vie de Yambo en sanctionnant dramatiquement la sortie de l'enfance.

9 Le parcours de Yambo dans cette histoire est un itinéraire dans l'encyclopédie visuelle du temps, que le lecteur est invité à reproduire en parcourant, le long des pages du livre, les reproductions des images d'une époque saisie surtout à travers sa culture 
populaire: des bandes dessinées surtout, depuis le mythique Flash Gordon, a Cino et Franco, l'Uomo Mascherato, Dick Tracy, Fantomas, l'Aventureux. Vient ensuite la littérature pour enfants avec les couvertures de la Bibliothèque pour mes enfants, les romans de Salgari, le courrier des petits, et aussi les chansons, les affiches publicitaires, les revues, les timbres, les objets, les images des jeunes fascistes et des paquets de cigarettes Fez.

10 Il est significatif que la reconstruction de l'encyclopédie culturelle de cette époque historique soit une grande fresque visuelle. Le choix de recourir à l'accumulation des images est étroitement lié au thème de la mémoire et au rôle à part qu'y jouent les sens. Souvent Eco a soutenu qu'une grammaire sensorielle différente régule nos souvenirs, assignant la primauté du visuel sur tous les autres sens: il est très difficile de se souvenir d'une saveur ou d'une odeur en tant que telle, mais il est beaucoup plus facile de se souvenir d'une image. Je ne sais s'il en est vraiment ainsi, mais Yambo se fie surtout à la vue pour réactiver sa mémoire perdue.

11 Mais quelles sont les images dont se sert Yambo? Dans le roman, il y a très peu de photos, quelques reproductions de pages de journaux, rares photos documentaires, quelques images cinématographiques. Mais, plus important encore, toutes les images appartiennent à l'encyclopédie d'une époque, ce sont des images d'une culture, non d'une histoire individuelle. Ce sont les bandes dessinées, les illustrations des livres et des journaux, les affiches, les chansons; s'il s'agit de photos, ce sont des photos "publiques ", qui renvoient au cinéma ou à l'actualité, et qui ne rappellent guère une expérience personnelle, spécifique, un champ subjectif, particulier et unique. Loana est un "roman illustré », comme il a été dit dans le sous-titre de la couverture de l'édition italienne, et illustré par toute la culture d'une époque. Et c'est encore une fois le pouvoir de l'encyclopédie qui dresse son réseau coloré et changeant de relations dans toutes les directions, suggérant des combinaisons et des parcours, même impensables entre les nœuds du réseau, mais qui renvoient toujours à un univers public et partagé de signifiés (au moins pour ceux qui appartiennent à la génération de Yambo et d'Eco).

12 Nous pourrions dire que La Mystérieuse flamme de la reine Loana est un roman d'images collectives, non individuelles, et c'est à travers cela que la structure visuelle du texte reproduit, en une sorte de mise en abîme expressive, sa propre structure narrative: l'histoire qui s'y raconte est précisément la perte de la subjectivité singulière dans celle culturelle.

13 Ceci, à une seule et importante exception. A la page 298 de l'édition française figure une petite photo en noir et blanc, une photo instantanée, un peu floue, qui représente deux enfants, un garçon plus grand qui se penche, légèrement embarrassé, pendant qu'une enfant un peu plus petite l'embrasse avec transport sur la joue. C'est la seule image "privée » de tout le roman, qui renvoie au monde individuel du protagoniste et non à celui encyclopédique, et c'est justement une photographie. Les photos ont parmi toutes les images un statut assez particulier : elles ne représentent pas en fait leur objet par voie de similitude formelle, mais en sont une vraie et propre trace physique. Elles témoignent, pour le dire avec Barthes (1980), d'une présence produite, points d'ancrage de l'existence individuelle: cet enfant, c'était moi, la photographie représente et garantit en même temps la continuité d'une singularité. Et c'est cela le punctum de la photographie.

14 Yambo sait que cette photo est la sienne, elle le montre lui-même à côté de la petite sœur, mais il ne s'y reconnaît pas, il n'arrive pas à la sentir comme part de sa propre 
histoire personnelle et la regarde comme une image d'un film, ou pourrions-nous dire, il la regarde encore avec un œil encyclopédique et non individuel.

15 Cette photo introduit une seconde aporie que la théorie ne peut, et peut être même ne doit, affronter et qui met en jeu, cette fois, le rapport complexe entre texte, auteur et lecteur. Le plus suspicieux des lecteurs pourrait s'interroger sur cette intrigante ressemblance entre ce petit garçon en culotte courte et notre célèbre auteur empirique. Les plus familiers avec lui savent qu'en effet c'est la photo de l'auteur enfant. Pourquoi donc cette photo, qui sanctionne l'irruption d'un élément autobiographique aussi concis à l'intérieur du monde fictionnel du romancier?

16 Nous savons très bien que tout biographisme exercé sur la figure de l'auteur empirique est un parcours critique impossible, et même d'une certaine façon interdit. Les histoires et les personnages que nous rencontrerons en ouvrant les pages d'une œuvre narrative sont irrémédiablement, je dirai presque ontologiquement, séparés et lointains de la personne réelle qui les a imaginés et écrits. Le monde raconté et le monde de celui qui raconte sont deux univers incommensurables qui ne peuvent se rencontrer parce que relevant de deux statuts différents de la réalité.

17 Cette impossibilité, Umberto Eco l'a écrite et théorisée dans toute sa production critique, insistant sur la distance indispensable que nous devons toujours mettre entre l'auteur et le lecteur empiriques, les personnes réelles qui produisent et lisent les textes, et ces « simulacres » qui sont l'auteur et le lecteur Modèles, pures stratégies qui existent seulement en tant que figures textuelles. L'Auteur Modèle, en tant que stratégie d'écriture, n'a aucun lien direct avec l'encombrante subjectivité de l'auteur empirique, dont l'existence et la mémoire se situent dans un hors texte inaccessible; la seule subjectivité qu'un texte peut rendre intelligible est celle inscrite dans ses formes textuelles. Que toute tentative d'en tirer plus soit illicite, le même Eco l'a plusieurs fois souligné, et pas que dans son œuvre théorique. Après la sortie du Nom de la rose, à ceux qui lui demandaient à quel personnage il s'identifiait le plus et où dans le roman sa subjectivité individuelle pouvait surtout être située, Eco répondit avec brio : «le sujet est dans les adverbes", une affirmation qui, de déclaration poétique, pourrait aussi valoir pour la théorie sémiotique d'Eco où également «le sujet est dans les adverbes », dissous dans le réseau des renvois encyclopédiques.

18 Mais pourquoi alors notre auteur a choisi cette photo précisément, sa photo, et non une quelconque photo d'un quelconque garçon de l'époque ? En jouant sciemment ainsi sur les marges ambigües entre son propre moi et celui des autres personnages, Eco rouvre cet espace mystérieux où la subjectivité individuelle de qui écrit émerge dans l'écriture.

19 Revenons ici au point d'où nous étions partis, le rapport complexe entre théorie et récit dans l'œuvre d'Eco, en posant de nouveau la question de comment les romans peuvent éclairer des points critiques que la théorie ne peut affronter. A ce stade, peutêtre, nous pouvons avancer une hypothèse: cet indicible élément théorique que raconte La Mystérieuse flamme de la reine Loana est vraiment le mystère de l'individuel, la composante la plus inexprimable de la subjectivité devant laquelle la théorie se retire, mais même la narration en perd ses mots, après l'avoir évoquée.

20 Durant tout le cours du roman, Yambo cherchera l'accès mystérieux à sa propre histoire individuelle, qu'assume symboliquement le visage de Lila, l'amour perdu. Mais le secret de l'individuel semble vraiment être l'extrême indicible : comme pour Martin Eden, on peut savoir seulement au moment où l'on cesse de savoir. Et même pour 
Yambo, à l'instant même où le visage resplendissant de l'être aimé commence à se révéler, donnant enfin sens et paix à sa quête fébrile, la vision s'éloigne pour toujours, la fumée l'enveloppe et le soleil se fait noir.

\section{BIBLIOGRAPHY}

Barthes, R. 1980, La chambre claire. Note sur la photographie, Paris : Gallimard-Seuil.

Eco, U.

1975 Trattato di semiotica generale, Milano : Bompiani.

1984 Semiotica e filosofia del linguaggio, Milano : Bompiani

Greimas, A. J. - Courtés, J.

1979 Sémiotique. Dictionnaire raisonné de la théorie du langage, Paris : Hachette

Paolucci, C. 2010 Strutturalismo e interpretazione, Milano : Bompiani

Violi, P.

2009 " "The subject is in the adverbs" The role of the subject in Eco's semiotics ", in P. Bondanella (ed.), New Essays on Umberto Eco, Cambridge : Cambridge University Press : 113-126.

2017 « Encyclopedia : criticality and actuality », in Randall E. Auxier and Sara Beardsworth eds., The Philosophy of Umberto Eco, The Library of Living Philosophers, Volume XXXV, Chicago, IL : Open Court Publishing Company.

\section{NOTES}

1. Sur ce point voir Violi (2009) Violi (2017) et Paolucci (2010).

\section{ABSTRACTS}

The aim of this text is to discuss some problematic aspects of Umberto Eco's theoretic elaboration, in particular the notions of subjectivity and individuality, rereading his more autobiographic novel The mysterious Flame of Queen Loana. The novel is paralleled with Eco's theory of subjectivity, showing how some problematic issues at the theoretical level are dealt with only in his fictional writing.

L'objet de cette étude est de discuter la conception d'Umberto Eco de la subjectivité à travers son roman, le plus autobiographique, La Mystérieuse Flamme de la reine Loana et de montrer comment 
cette conception est profondément inscrite dans l'importance fondamentale et déterminante qu'il donne aux signes dans leur formation du sens et de cette même subjectivité.

INDEX

Mots-clés: subjectivité, individu, semiosis, Loana, autobiographie

Keywords: subjectivity, subject, semiosis, Loana, autobiography

\section{AUTHORS}

\section{PATRIZIA VIOLI}

Université de Bologne 